\title{
Enxerto espesso versus técnica de duplo feixe na reconstrução do ligamento cruzado posterior: Estudo experimental biomecânico com cadáveres*
}

\section{Thick Graft versus Double-bundle Technique on Posterior Cruciate Ligament Reconstruction: Experimental Biomechanical Study with Cadavers}

João Alberto Ramos Maradei-Pereira ${ }^{1,2,3(0)}$ Alexandre Estevão Vamos Kokron ${ }^{3}$ César Augusto Martins Pereira ${ }^{3}$ Marco Martins Amatuzzi ${ }^{3}$

\footnotetext{
${ }^{1}$ Faculdade de Medicina, Universidade Federal do Pará, Belém, PA, Brasil

${ }^{2}$ Clínica dos Acidentados, Hospital Maradei, Belém, PA, Brasil

${ }^{3}$ Laboratório de Investigação Médica (LIM-41), Instituto de Ortopedia e Traumatologia, Hospital das Clinicas, Faculdade de Medicina, Universidade de Sao Paulo, São Paulo, SP, Brasil
}

Endereço para correspondência João Alberto Ramos Maradei-Pereira, MD, MSc, Faculdade de Medicina, Universidade Federal do Pará, Praça Camilo Salgado, 01, Umarizal, Belém, PA, 66050-060 Brazil (e-mail: jamaradei@ufpa.br).

Rev Bras Ortop 2019;54:531-539.

\begin{abstract}
Resumo
Palavras-Chave

- traumatismos do joelho

- ligamento cruzado posterior

- fenômenos biomecânicos

- cadáver

- reconstrução do ligamento cruzado posterior

Objetivo Avaliar o efeito biomecânico da espessura do enxerto em comparação com a técnica do duplo feixe na reconstrução do ligamento cruzado posterior (LCP) em joelhos de cadáveres humanos.

Métodos Um total de 9 joelhos de cadáveres humanos foram testados em 5 condições: joelho intacto (INT); reconstrução com um único feixe com tendão de quadríceps de $10 \mathrm{~mm}$ ( $\operatorname{Rec} 1$ ); reconstrução com duplo feixe com um tendão de quadríceps de $10 \mathrm{~mm}$ para o feixe anterolateral e um tendão duplo do semitendíneo de $7 \mathrm{~mm}$ para o feixe póstero-medial (Rec 2); reconstrução com um único feixe mais espesso, usando um tendão de quadríceps de $10 \mathrm{~mm}$ mais o tendão duplo do semitendíneo de $7 \mathrm{~mm}$ (Rec M); e joelho com lesão isolada do LCP (Lesionado). O limite do deslocamento posterior da tíbia (LDPT) foi medido em resposta a uma carga tibial posterior de $134 \mathrm{~N}$ a $0^{\circ}, 30^{\circ}, 60^{\circ}$ e $90^{\circ}$ de flexão do joelho.

Resultados O LDPT das técnicas Rec 2 e Rec M foi sempre significativamente menor (melhor estabilidade) do que o LDPT da Rec 1. O LDPT da Rec M foi significativamente menor do que o LDPT da $\operatorname{Rec} 2$ a $60^{\circ}(p=0,005)$ e a $90^{\circ}(p=0,001)$.

Conclusões $\mathrm{O}$ aumento da espessura do enxerto na reconstrução das lesões isoladas do LCP melhora significativamente a estabilidade, enquanto a divisão do enxerto para
\end{abstract}

\footnotetext{
Estudo desenvolvido no Laboratório de Investigação Médica (LIM-41), Instituto de Ortopedia e Traumatologia, Hospital das Clínicas HCFMUSP, Faculdade de Medicina, Universidade de São Paulo, São Paulo, SP, Brasil. Publicado Originalmente por Elsevier Editora Ltda.
}

recebido

29 de Setembro de 2017

aceito

14 de Dezembro de 2017
DOI https://doi.org/

10.1016/j.rboe.2017.12.010. ISSN 0102-3616.
Copyright $\odot 2019$ by Sociedade Brasileira License terms de Ortopedia e Traumatologia. Published by Thieme Revinter Publicações Ltda, Rio de Janeiro, Brazil 
Abstract

\section{Keywords}

- knee injuries

- posterior cruciate ligament

- biomechanical phenomena

- cadaver

- posterior cruciate ligament reconstruction reconstruir os dois feixes piora a estabilidade da reconstrução a $60^{\circ}$ e $90^{\circ}$ de flexão do joelho. As descobertas do presente estudo sugerem que a estabilidade da reconstrução do LCP pode ser melhorada com o uso de enxertos mais espessos em uma técnica de feixe único, em lugar da reconstrução de duplo feixe.

Objective To evaluate the biomechanical effect of graft thickness compared with the double-bundle technique on posterior cruciate ligament $(\mathrm{PCL})$ reconstruction in human cadaveric knees.

Methods A total of 9 human cadaveric knees were tested in 5 conditions: intact knee (INT); single-bundle reconstruction with a $10-\mathrm{mm}$ quadriceps tendon (SB); doublebundle reconstruction with a $10-\mathrm{mm}$ quadriceps tendon for the anterolateral bundle and a 7-mm doubled semitendinosus tendon for the posteromedial bundle (DB); singlebundle reconstruction with a 10-mm quadriceps tendon plus a 7-mm doubled semitendinosus tendon (SBT); and PCL-deficient (NoPCL). The posterior tibial translation (PTT) was measured in response to a $134 \mathrm{~N}$ posterior tibial load at $0^{\circ}, 30^{\circ}, 60^{\circ}$, and $90^{\circ}$ of knee flexion.

Results The PTT of the DB and SBT techniques was always significantly lower (better stability) than the PTT of the SB technique. The PTT of the SBT technique was significantly lower than the PTT of the DB technique at $60^{\circ}(p=0.005)$ and $90^{\circ}(p=0.001)$.

Conclusions Graft enlargement improves knee stability in isolated PCL reconstructions, whereas the graft division in the two-bundle technique worsens this stability at $60^{\circ}$ and $90^{\circ}$ of knee flexion. The findings of the present study suggest that knee stability in PCL reconstructions may be improved with the use of thicker grafts in the single-bundle technique rather than performing a double-bundle reconstruction.

\section{Introdução}

O tratamento ideal das lesões do ligamento cruzado posterior (LCP) ainda não está definido. Embora haja consenso na literatura sobre a necessidade de tratamento cirúrgico na presença de outras lesões ligamentares do joelho, ${ }^{1,2}$ a controvérsia permanece nas lesões isoladas do LCP. Alguns autores defendem o tratamento conservador nesses casos. ${ }^{3,4}$ Outros, no entanto, enfatizam que o joelho com lesão do LCP desenvolve alterações degenerativas que se agravam com o tempo., 5, O melhor entendimento da anatomia e da biomecânica do ligamento permitiu melhorias nos resultados das reconstruções do LCP, mas ainda não existe uma técnica ideal. ${ }^{7,8}$

Com base em estudos anatômicos e biomecânicos, alguns autores sugerem que a reconstrução com dois feixes e dois túneis femorais mimetiza melhor a anatomia e a função do joelho normal. ${ }^{1,9}$ No entanto, há um viés nos testes laboratoriais que mostram a superioridade dessas reconstruções de feixe duplo ${ }^{10,11}$ decorrentes da falta de uniformidade na espessura dos enxertos usados nos dois grupos comparados. Nestes estudos, os autores utilizam um volume maior de enxerto na reconstrução de dois feixes do que na técnica com um feixe. Ao utilizar enxertos da mesma espessura em ambos os grupos, outros autores não observaram diferença significativa entre as técnicas. ${ }^{12,13}$ Sobre isso, formulou-se a questão que motivou este trabalho: a restauração bem-sucedida da estabilidade do joelho alcançada com a técnica do feixe duplo deve-se à reconstrução do segundo feixe ou ao maior volume de enxerto? O objetivo deste estudo foi, portanto, avaliar o efeito biomecânico da espessura do enxerto comparado com a técnica de duplo feixe na reconstrução do LCP em joelhos de cadáveres humanos.

\section{Métodos}

Este estudo foi aprovado pelo Comitê de Ética em Pesquisa da instituição responsável (número de protocolo 391/02).

Os joelhos utilizados neste estudo foram obtidos de nove cadáveres frescos do serviço de necropsia de um hospital universitário de grande porte no Brasil. A idade média dos cadáveres foi de 52 anos, variando de 43 a 70 anos. Sete eram do sexo masculino.

No serviço de necropsia, realizou-se uma artrotomia parapatelar medial e uma vertical posterior, para inspeção articular e exclusão de qualquer espécime com lesão ligamentar ou doença articular degenerativa. Uma vez que os espécimes foram considerados elegíveis para o estudo, as artrotomias foram fechadas com um fio de nylon de 2-0. 0 joelho foi então retirado do cadáver e congelado a $-15^{\circ} \mathrm{C}$.

\section{Preparação das amostras}

$\mathrm{O}$ joelho foi descongelado durante a noite à temperatura ambiente. Em seguida, foi dissecado, teve a pele removida, poupando todos os ligamentos do joelho, incluindo os da articulação tibiofibular proximal, tendão e músculo poplíteo, membrana interóssea, cápsula articular e mecanismo extensor. A fíbula foi estabilizada na tíbia com um parafuso cortical 
de $4,5 \mathrm{~mm}$, conforme recomendado, ${ }^{10,12,14}$ e seccionada a uma distância de $2 \mathrm{~cm}$ distalmente ao parafuso.

Os enxertos obtidos do joelho a ser testado foram um tendão do quadríceps de $10 \mathrm{~mm}$ e um semitendíneo duplo. Eles foram submetidos a uma tensão constante de $20 \mathrm{~N}$ por 20 minutos e utilizados em reconstruções após esse período.

\section{Protocolo de teste}

Os ensaios mecânicos foram realizados na máquina de testes eletromecânica Kratos K5002 (Kratos Equipamentos Industriais, Cotia, SP, Brasil), com uma célula de carga eletrônica de $100 \mathrm{kgf}$ conectada a um computador equipado com um sistema de aquisição de dados ADS 2000 (Lynx Tecnologia Eletrônica Ltda., São Paulo, SP, Brasil). Para iniciar os testes, uma garra metálica foi fixada à metáfise e diáfise distal do fêmur, e outra à diáfise proximal da tíbia. Elas permaneceram nesta posição firmemente fixadas durante todos os testes, e até durante os procedimentos cirúrgicos. $\mathrm{O}$ joelho com as garras foi fixado na máquina de testes ( - Fig. 1), e esta conectada ao computador. Para cada avaliação, o joelho foi testado em extensão, e a $30^{\circ}, 60^{\circ}$ e $90^{\circ}$ de flexão.

Durante os testes, os movimentos do fêmur na direção superior e inferior em relação à tíbia foram feitos pela máquina Kratos a uma velocidade constante de $20 \mathrm{~mm} /$ min, correspondendo ao teste de gaveta anterior e posterior, respectivamente. A célula de carga foi localizada na metáfise femoral distal. Os resultados dos testes (força aplicada à tíbia e seu deslocamento desde o início do teste) eram imediatamente transferidos ao computador. Para cada condição de avaliação, a peça foi submetida a três ciclos consecutivos de aplicação de força pela máquina de teste. Inicialmente, a carga foi aplicada na direção do fêmur para obter uma translação anterior da tíbia de $2 \mathrm{~mm}$. Depois, foi feita no sentido reverso (gaveta posterior) com força equivalente a $134 \mathrm{~N}$, como descrito por alguns autores. ${ }^{11,15,16}$
A frouxidão de cada joelho foi inicialmente testada com ligamentos intactos (INTs). Em seguida, o LCP foi removido, e cada joelho foi submetido sucessivamente a três tipos diferentes de reconstrução, descritos detalhadamente a seguir. A frouxidão foi testada após cada reconstrução. Ao final, os testes foram realizados no joelho sem o LCP original e sem reconstrução (Lesionado) (-Fig.2).

\section{Técnicas cirúrgicas}

Reconstrução de feixe único com tendão de $10 \mathrm{~mm}$ do quadríceps ( $\operatorname{Rec} 1$ )

Depois de ser testado com o LCP intacto, o joelho foi removido da máquina de testes. Todas as técnicas cirúrgicas foram realizadas por meio de artrotomia parapatelar medial e por uma pequena artrotomia longitudinal posterior. Desta forma, o LCP foi completamente removido, incluindo os ligamentos meniscofemorais, quando presentes, e poupando os demais ligamentos do joelho. 0 túnel tibial foi feito com $10 \mathrm{~mm}$ de diâmetro, desde a superfície anteromedial da tíbia até o centro da inserção tibial do LCP removido. O túnel femoral anterolateral (AL) de $10 \mathrm{~mm}$ foi perfurado centralizado a $7 \mathrm{~mm}$ da borda da cartilagem na posição de 1 hora, em uma direção paralela ao teto da fossa. 0 enxerto de tendão do quadríceps foi passado pelos túneis tibial e femoral, com o bloco ósseo localizado no túnel femoral. Foi então fixado ao fêmur, sendo amarrado em torno de um parafuso cortical de $4,5 \mathrm{~mm}$ com arruela.

Após a sutura da cápsula articular, o joelho foi reposicionado na máquina de testes a $90^{\circ}$ de flexão e submetido a uma gaveta anterior com carga de $134 \mathrm{~N}$. Neste momento, o enxerto foi tensionado (Tensionador Digital, LIM-41, São Paulo, SP, Brasil) ( - Fig. 3).Quando a tensão desejada (88 N) foi alcançada, o enxerto foi fixado à tíbia por meio do travamento dos dois fios de poliéster $n$. 5 entre 2 plaquetas metálicas e 1 parafuso (-Fig.4).

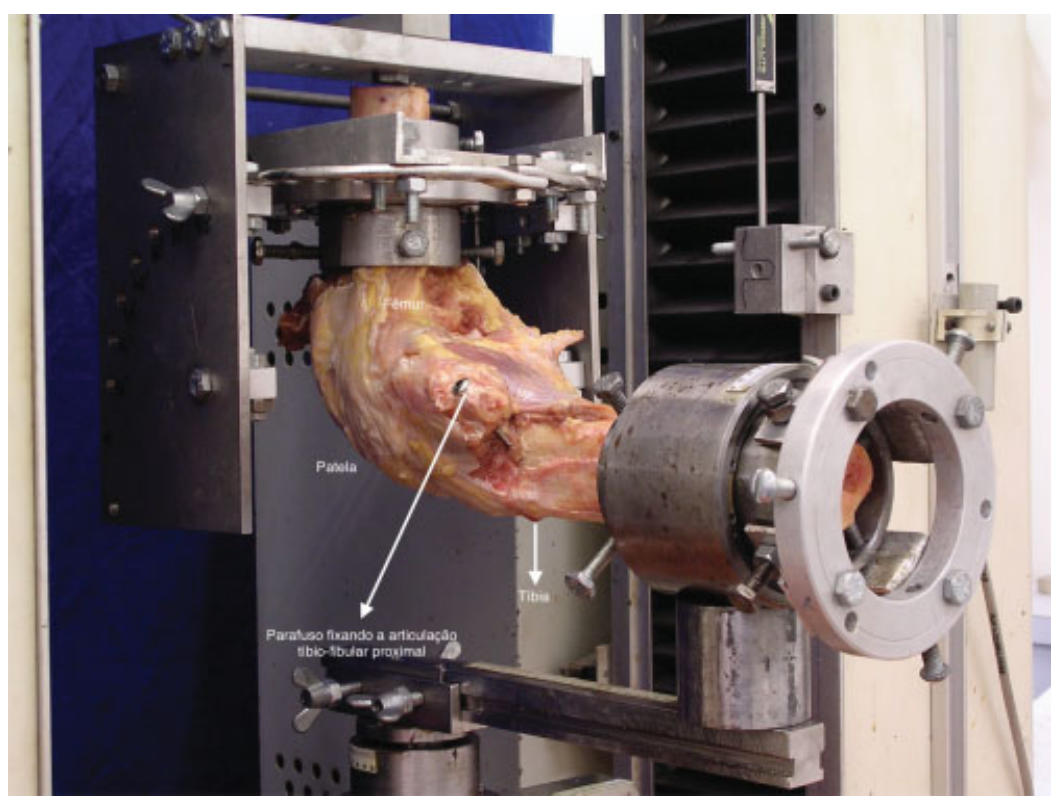

Fig. 1 Joelho posicionado a $90^{\circ}$ de flexão, fixado à máquina de teste. A tíbia permaneceu horizontalmente com a margem anterior voltada para o solo. A máquina realizava a elevação ou descida do fêmur em relação à tíbia, correspondendo, respectivamente, ao movimento de gaveta anterior e posterior. 
534 Enxerto espesso versus técnica de duplo feixe na reconstrução do ligamento cruzado posterior Maradei-Pereira et al.

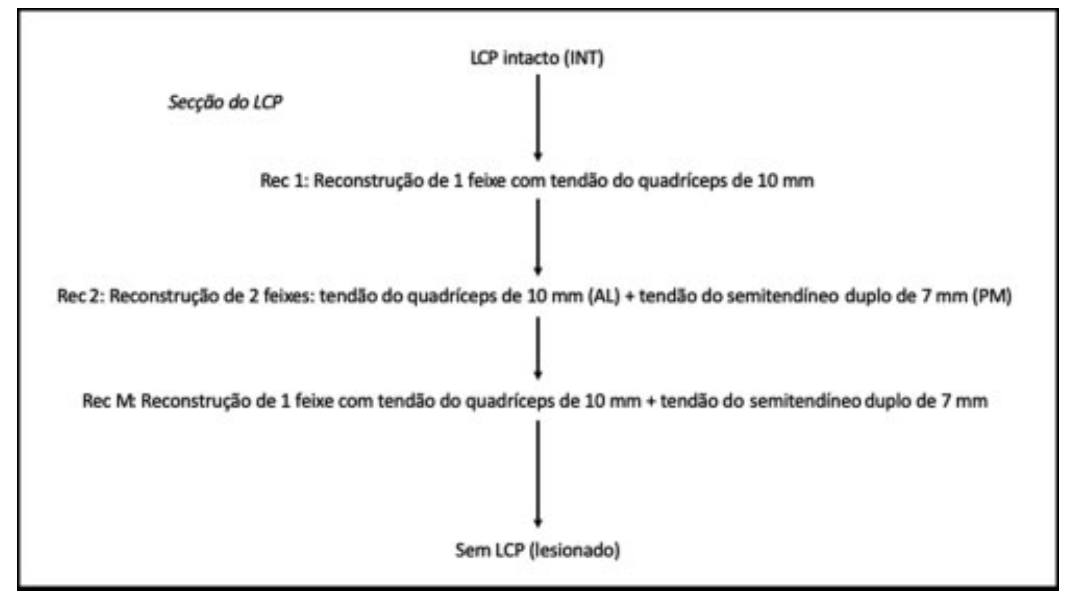

Fig. 2 Sequência das condições de teste.

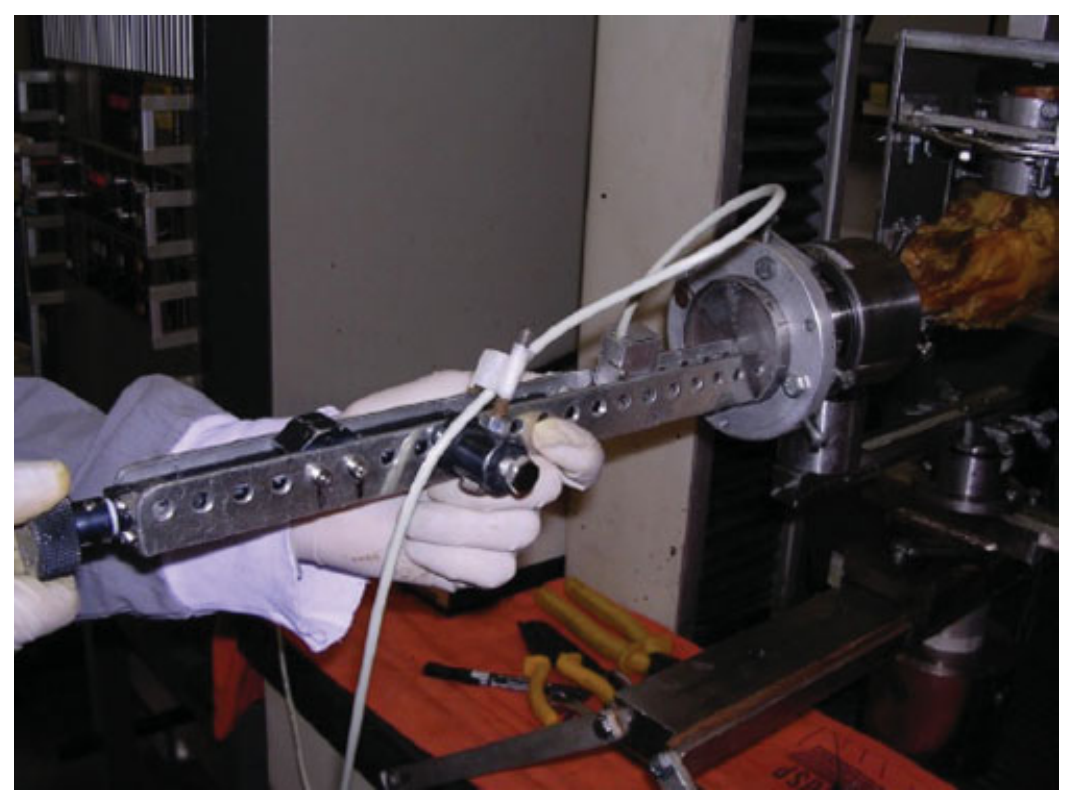

Fig. 3 Tensionamento do enxerto monitorado pelo dinamômetro.

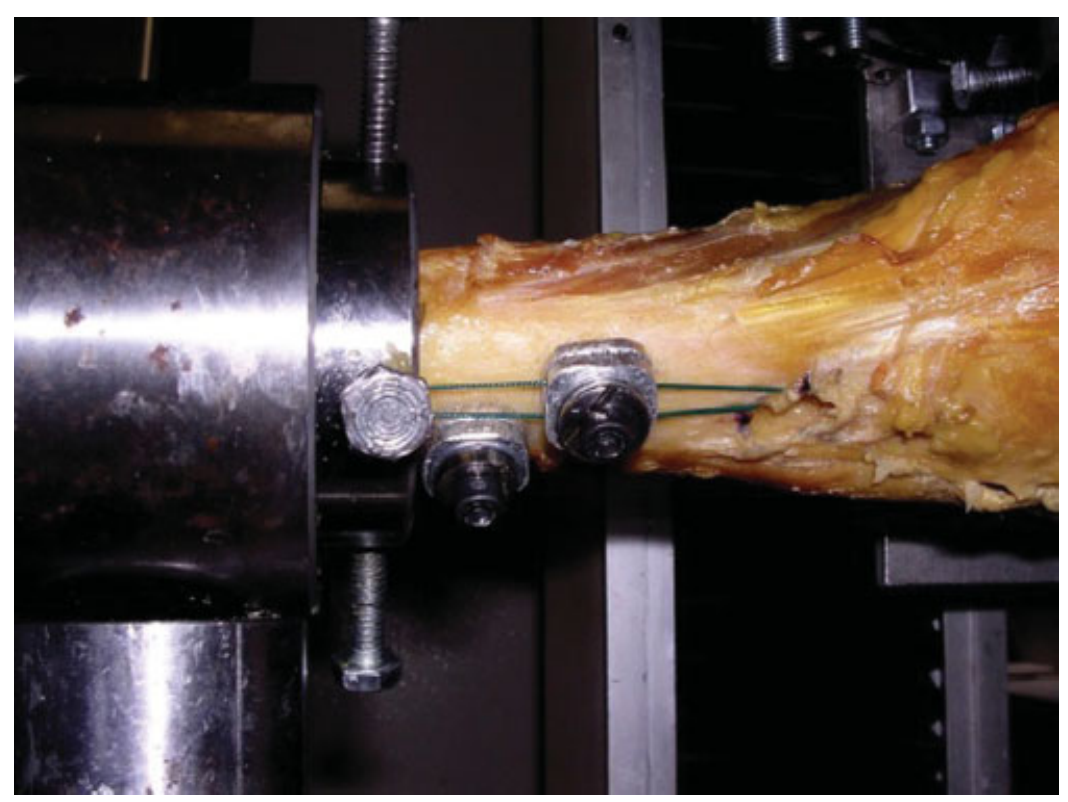

Fig. 4 O dispositivo de fixação tibial. Os fios de poliéster do enxerto foram bloqueados entre duas plaquetas. 


\section{Reconstrução de feixe duplo $(\operatorname{Rec} 2)$}

Depois de testar a reconstrução de feixe único $(\operatorname{Rec} 1)$, o joelho foi removido da máquina de testes. As suturas retiradas das artrotomias, assim como o enxerto do túnel tibial. Este túnel foi aumentado em espessura com uma broca de $12 \mathrm{~mm}$ de diâmetro.

$O$ enxerto foi mantido no túnel femoral AL enquanto o segundo túnel (póstero-medial, PM) foi confeccionado. Este túnel foi centrado na posição de $2 \mathrm{~h} 30$, a $14 \mathrm{~mm}$ da borda da cartilagem (direção distal-proximal) e $9 \mathrm{~mm}$ posterior ao centro do túnel $\mathrm{AL}$ (direção ântero-posterior).

Este túnel femoral PM foi confeccionado com broca de $7 \mathrm{~mm}$ de diâmetro. $O$ enxerto do tendão semitendíneo duplo foi passado através dos túneis tibial e PM femoral e fixo ao fêmur, amarrando-o em torno de um segundo parafuso cortical com arruela (4,5 mm de diâmetro). Em seguida, o enxerto do quadríceps foi novamente passado pelo túnel tibial em posição anterior ao semitendíneo.

Após as suturas das artrotomias, o joelho foi reposicionado na máquina de testes a $90^{\circ}$ de flexão e submetido a uma gaveta anterior com carga de $134 \mathrm{~N}$. O enxerto do quadríceps foi então fixado com $88 \mathrm{~N}$ de tensão. $O$ joelho foi retornado à posição 0 e posicionado em $0^{\circ}$ de flexão. Foi novamente submetido a uma gaveta anterior de $134 \mathrm{~N}$, e o enxerto semitendíneo tensionado a $67 \mathrm{~N}$ e fixado com um segundo dispositivo tibial (semelhante ao usado para o quadríceps, -Figs. 3 e 4). 0 joelho foi então retornado à posição 0 e submetido aos testes de frouxidão a $0^{\circ}, 30^{\circ}, 60^{\circ}$ e $90^{\circ}$ de flexão.

Reconstrução de feixe único com tendão do quadríceps e do semitendíneo (Rec M)

Após os testes da reconstrução de feixe duplo (Rec 2 ), ambos os enxertos foram liberados do fêmur e retirados de seus respectivos túneis, exceto do tibial.

O túnel femoral AL foi aumentado em espessura com uma broca de $12 \mathrm{~mm}$. Ambos os enxertos foram então passados através deste túnel e fixados ao fêmur, sendo amarrados ao mesmo parafuso bicortical previamente usado para fixar apenas o enxerto do quadríceps. O parafuso cortical que fixava o semitendíneo no túnel femoral PM foi então removido.

A inserção tibial dos enxertos foi solta, a artrotomia medial fechada, e o joelho reposicionado na máquina de teste a $90^{\circ}$ de flexão. $O$ espécime foi submetido a uma gaveta anterior com carga de $134 \mathrm{~N}$, os enxertos tensionados ambos a $90^{\circ}$ : o quadríceps com $88 \mathrm{~N}$, e o semitendíneo com $67 \mathrm{~N}$, e fixados à tíbia, cada um em seu próprio dispositivo de fixação tibial (conforme descrito anteriormente). 0 joelho foi então retornado à posição 0 e testado a $0^{\circ}, 30^{\circ}, 60^{\circ}$ e $90^{\circ}$ de flexão.

\section{Cálculo do tamanho da amostra}

Um estudo bastante similar ${ }^{13}$ avaliou o valor da translação posterior da tíbia, e obteve uma diferença aproximada de 7,5 mm entre os joelhos, comparando as situações reconstruída e lesionada na angulação de flexão intermediária. A maior variabilidade encontrada durante todos os ângulos de flexão foi $\sim 5,66 \mathrm{~mm}$ (desvio padrão $[\mathrm{DP}]=5.66$ ). Com base nesse estudo e com o objetivo de encontrar um resultado semelhante, com $80 \%$ de poder e intervalo de confiança de 95\% (IC95\%), a amostra necessária para o presente estudo foi calculada como 9 joelhos submetidos a todas as 5 situações (INT, Rec 1, Rec 2, Rec M e Lesionado).

\section{Coleta de dados e análise estatística}

Os dados foram representados pelas seguintes medidas descritivas: média, DP, e limites superior e inferior do IC $95 \%$. O nível de significância estatística $(p)$ de 0,05 (5\%) foi adotado. Níveis descritivos abaixo deste valor foram considerados significativos. Com base no gráfico da força aplicada pela translação da tíbia em todos os ângulos medidos, o limite de deslocamento posterior da tíbia (LDPT) foi determinado. Foi medido em milímetros e definido como a maior translação posterior da tíbia.

A análise de variância (ANOVA, em inglês) para medidas repetidas foi utilizada, com dois fatores: condições de teste nas 5 situações (INT, Rec 1 , Rec 2 , Rec M e Lesionado) e grau de flexão do joelho com quatro níveis $\left(0^{\circ}, 30^{\circ}, 60^{\circ}\right.$ e $\left.90^{\circ}\right)$. 0 teste de comparação múltipla de Newman-Keuls foi usado para encontrar diferenças entre as técnicas e entre os graus de flexão do joelho.

\section{Resultados}

\section{Limite de deslocamento posterior da tíbia}

Os dados de LDPT (média, DP, e ICs95\% inferiores e superiores) das 5 condições de teste em cada um dos 4 ângulos testados são mostrados na - Tabela 1, e sua representação gráfica, na - Fig. 5. A comparação entre as cinco condições de teste em cada ângulo de flexão do joelho é mostrada nas

\section{- Tabelas 2-5.}

O LDPT da Rec 1 foi estatisticamente maior (pior frouxidão) do que o do joelho INT em todos os ângulos testados $\left(p=0,022\right.$ a $0^{\circ} ; p<0,001$ a $30^{\circ}, 60^{\circ}$ e $\left.90^{\circ}\right)$. A Rec 2 apresentou LDPT estatisticamente similar à do joelho INT a $0^{\circ}(p=0,18) \mathrm{e}$ a $30^{\circ}(p=0,085)$. A Rec M foi similar ao joelho INT a $0^{\circ}$ $(p=0,803)$, a $30^{\circ}(p=0,98)$ e a $60^{\circ}(p=0,116)$ de flexão.

A reconstrução de feixe único com enxerto mais espesso (Rec M) apresentou valores mais baixos de LDPT (melhor frouxidão) do que a $\operatorname{Rec} 2$ a $60^{\circ}(p=0,005)$ e a $90^{\circ}(p=0,001)$. A $90^{\circ}$, todas as reconstruções do LCP apresentaram LDPT estatisticamente diferente em relação ao joelho INT.

\section{Discussão}

Uma recente revisão sistemática ${ }^{17}$ da literatura analisou os resultados de estudos comparando o uso de um ou dois feixes na reconstrução do LCP. ${ }^{17} \mathrm{~A}$ revisão conseguiu reunir apenas 11 estudos clínicos até o momento, mostrando que ainda há uma lacuna na literatura sobre esse tema. Alguns autores relatam resultados mais fisiológicos e melhor frouxidão com a reconstrução de dois feixes. ${ }^{2,10,11,18-25}$ Outros, no entanto, não conseguiram demonstrar a superioridade de uma técnica sobre a outra (um feixe versus dois feixes) ${ }^{12,13,26-29}$ Este cenário nos fez pensar se a comparação deveria ser feita entre um e dois feixes ou se, por outro lado, há um problema com o 
Tabela 1 Limite de deslocamento posterior da tíbia, em milímetros, por condições de teste e ângulo de flexão do joelho. Média, desvio padrão (entre parênteses) e limites do intervalo de confiança de 95\% (entre colchetes)

\begin{tabular}{|l|l|l|l|l|l|}
\hline \multirow{2}{*}{$\begin{array}{l}\text { Ângulo de } \\
\text { flexão }\end{array}$} & & & LDPT $(\mathbf{m m})$ & & \\
\cline { 2 - 6 } & Intacto & Lesionado & $\operatorname{Rec} 1$ & $\operatorname{Rec} \mathbf{2}$ & Rec M \\
\hline $0^{\circ}$ & $9,48(1,81)$ & $12,30(2,72)$ & $10,46(2,12)$ & $8,83(1,60)$ & $9,55(1,76)$ \\
& {$[8,08-10,86]$} & {$[10,21-14,40]$} & {$[8,83-12,08]$} & {$[7,61-10,06]$} & {$[8,19-10,90]$} \\
\hline $30^{\circ}$ & $10,98(1,93)$ & $17,36(3,24)$ & $13,70(2,35)$ & $12,00(1,97)$ & $10,96(2,63)$ \\
& {$[9,49-12,46]$} & {$[14,87-19,85]$} & {$[11,89-15,51]$} & {$[10,49-13,52]$} & {$[8,93-12,98]$} \\
\hline $60^{\circ}$ & $8,92(1,24)$ & $17,87(3,01)$ & $12,60(1,97)$ & $11,18(1,82)$ & $9,87(2,06)$ \\
& {$[7,97-8,87]$} & {$[15,55-20,18]$} & {$[11,09-14,12]$} & {$[9,78-12,58]$} & {$[8,29-11,46]$} \\
\hline $90^{\circ}$ & $8,31(1,26)$ & $19,28(2,33)$ & $12,83(2,16)$ & $11,67(2,22)$ & $10,27(2,43)$ \\
& {$[7,34-9,28]$} & {$[17,49-21,07]$} & {$[11,17-14,49]$} & {$[9,96-13,37]$} & {$[8,40-12,14]$} \\
\hline
\end{tabular}

Abreviaturas: Intacto, ligamento cruzado posterior intacto; LDPT, limite de deslocamento posterior da tíbia; Lesionado, ligamento cruzado posterior lesionado; Rec 1 , reconstrução de feixe único com tendão do quadríceps de $10 \mathrm{~mm}$; Rec 2, reconstrução de duplo feixe com um tendão do quadríceps de $10 \mathrm{~mm}$ e um feixe de $7 \mathrm{~mm}$ do semitendíneo duplo; Rec M, reconstrução de feixe único com um tendão do quadríceps de $10 \mathrm{~mm}$ mais um feixe de $7 \mathrm{~mm}$ do semitendíneo duplo.

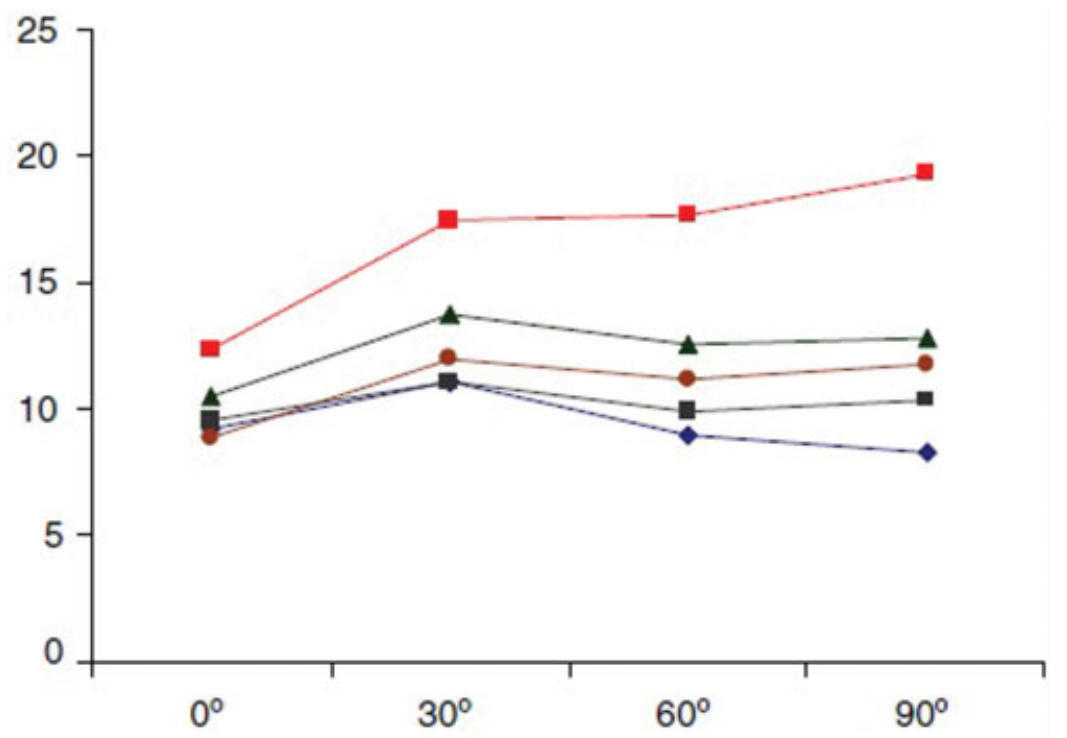

\section{Knee flexion angle}

Fig. 5 O limite de deslocamento posterior da tíbia (LDPT) de todas as condições de teste a cada ângulo de flexão: ligamento cruzado posterior (LCP) intacto (linha azul); LCP lesionado (linha vermelha); reconstrução de feixe único com tendão do quadríceps de $10 \mathrm{~mm}$ (linha verde); reconstrução de duplo feixe com um tendão do quadríceps de $10 \mathrm{~mm}$ para o feixe anterolateral e um feixe de $7 \mathrm{~mm}$ do semitendíneo duplo para o feixe póstero-medial (linha marrom); reconstrução de feixe único com um tendão do quadríceps de $10 \mathrm{~mm}$ mais um feixe de $7 \mathrm{~mm}$ do semitendíneo duplo (linha preta).

volume do enxerto, que seria mais bem resolvido com um único feixe mais espesso (e não com vários feixes). Em seguida, identificamos dois pontos-chave para um estudo mais aprofundado: a espessura do enxerto e o segundo túnel femoral. A influência desses dois fatores na frouxidão da reconstrução foi testada.

Aumentar a espessura do enxerto (adicionando o tendão semitendíneo duplo de $7 \mathrm{~mm}$ ao enxerto do quadríceps de $10 \mathrm{~mm}$ ) em nossas reconstruções de feixe único reduziu significativamente $(p<0,05)$ a translação posterior da tíbia em todos os ângulos testados. Escolhemos o posicionamento AL, não isométrico, para a inserção femoral em nossas reconstruções de feixe único, uma vez que o feixe anterolateral do LCP tem mais fibras e maior resistência final do que o
PM. Também, porque a reconstrução com o túnel femoral nesta posição resulta em maior estabilidade do que a obtida com outras técnicas (PM ou reconstruções isométricas)..$^{10,30}$ Nós reproduzimos a posição do túnel AL como feita no estudo de Kokron et al, ${ }^{12}$ a qual também corresponde à posição do túnel raso identificado como S1 no estudo de Mannor et al., ${ }^{31}$ e ao túnel AL em Harner et al ${ }^{11}$ e Bergfeld et al. ${ }^{13} \mathrm{O}$ valor de $88 \mathrm{~N}$ de tensionamento do enxerto a $90^{\circ}$ de flexão, com gaveta anterior de $134 \mathrm{~N}$, foi recomendado por Harner et al. ${ }^{11}$

A Rec 1 não foi capaz de restaurar a estabilidade do joelho em nenhum dos ângulos mensurados. 0 mesmo resultado foi alcançado por Kokron et a ${ }^{12}$ com o mesmo procedimento de Harner et al, ${ }^{11}$ com um enxerto de tendão de Aquiles de $10 \mathrm{~mm}$. Em ambos os estudos, o mesmo feixe $\mathrm{AL}$ não 
Tabela 2 Comparação dos níveis descritivos do deslocamento posterior da tíbia a $0^{\circ}$ de flexão entre as condições de teste

\begin{tabular}{|l|l|l|l|l|l|}
\hline & Intacto & Lesionado & $\operatorname{Rec} 1$ & $\operatorname{Rec} 2$ & $\operatorname{Rec} \mathbf{M}$ \\
\hline Intacto & - & $\mathrm{x}$ & $\mathrm{x}$ & $\mathrm{x}$ & $\mathrm{x}$ \\
\hline Lesionado & $p=0,001$ & - & $\mathrm{x}$ & $\mathrm{x}$ & $\mathrm{x}$ \\
\hline $\operatorname{Rec} 1$ & $p=0,022$ & $p=0,003$ & - & $\mathrm{x}$ & $\mathrm{x}$ \\
\hline $\operatorname{Rec} 2$ & $p=0,180$ & $p<0,001$ & $p=0,001$ & - & $\mathrm{x}$ \\
\hline $\operatorname{Rec} \mathrm{M}$ & $p=0,803$ & $p=0,006$ & $p=0,036$ & $p=0,183$ & - \\
\hline
\end{tabular}

Abreviaturas: Intacto, ligamento cruzado posterior intacto; Lesionado, ligamento cruzado posterior lesionado; Rec 1 , reconstrução de feixe único com tendão do quadríceps de $10 \mathrm{~mm}$; $\operatorname{Rec} 2$, reconstrução de duplo feixe com um tendão do quadríceps de $10 \mathrm{~mm}$ e um feixe de $7 \mathrm{~mm}$ do semitendíneo duplo; Rec M, reconstrução de feixe único com um tendão do quadríceps de $10 \mathrm{~mm}$ mais um feixe de $7 \mathrm{~mm}$ do semitendíneo duplo.

Tabela 3 Comparação dos níveis descritivos do deslocamento posterior da tíbia a $30^{\circ}$ de flexão entre as condições de teste

\begin{tabular}{|l|l|l|l|l|l|}
\hline & Intacto & Lesionado & $\operatorname{Rec} 1$ & $\operatorname{Rec} 2$ & $\operatorname{Rec} \mathbf{M}$ \\
\hline Intacto & - & $\mathrm{x}$ & $\mathrm{x}$ & $\mathrm{x}$ & $\mathrm{x}$ \\
\hline Lesionado & $p<0,001$ & - & $\mathrm{x}$ & $\mathrm{x}$ & $\mathrm{x}$ \\
\hline $\operatorname{Rec} 1$ & $p<0,001$ & $p=0,001$ & - & $\mathrm{x}$ & $\mathrm{x}$ \\
\hline $\operatorname{Rec} 2$ & $p=0,085$ & $p<0,001$ & $p=0,001$ & - & $\mathrm{x}$ \\
\hline $\operatorname{Rec} \mathrm{M}$ & $p=0,980$ & $p=0,001$ & $p=0,002$ & $p=0,140$ & - \\
\hline
\end{tabular}

Abreviaturas: Intacto, ligamento cruzado posterior intacto; Lesionado, ligamento cruzado posterior lesionado; Rec 1 , reconstrução de feixe único com tendão do quadríceps de $10 \mathrm{~mm}$; Rec 2 , reconstrução de duplo feixe com um tendão do quadríceps de $10 \mathrm{~mm}$ e um feixe de $7 \mathrm{~mm}$ do semitendíneo duplo; Rec $\mathrm{M}$, reconstrução de feixe único com um tendão do quadríceps de $10 \mathrm{~mm}$ mais um feixe de $7 \mathrm{~mm}$ do semitendíneo duplo.

Tabela 4 Comparação dos níveis descritivos do deslocamento posterior da tíbia a $60^{\circ}$ de flexão entre as condições de teste

\begin{tabular}{|l|l|l|l|l|l|}
\hline & Intacto & Lesionado & $\operatorname{Rec} 1$ & $\operatorname{Rec} 2$ & $\operatorname{Rec} \mathbf{M}$ \\
\hline Intacto & - & $\mathrm{x}$ & $\mathrm{x}$ & $\mathrm{x}$ & $\mathrm{x}$ \\
\hline Lesionado & $p<0,001$ & - & $\mathrm{x}$ & $\mathrm{x}$ & $\mathrm{x}$ \\
\hline $\operatorname{Rec} 1$ & $p<0,001$ & $p<0,001$ & - & $\mathrm{x}$ & $\mathrm{x}$ \\
\hline $\operatorname{Rec} 2$ & $p=0,001$ & $p<0,001$ & $p=0,003$ & - & $\mathrm{x}$ \\
\hline $\operatorname{Rec} \mathrm{M}$ & $p=0,116$ & $p<0,001$ & $p=0,001$ & $p=0,005$ & - \\
\hline
\end{tabular}

Abreviaturas: Intacto, ligamento cruzado posterior intacto; Lesionado, ligamento cruzado posterior lesionado; Rec 1, reconstrução de feixe único com tendão do quadríceps de $10 \mathrm{~mm}$; Rec 2, reconstrução de duplo feixe com um tendão do quadríceps de $10 \mathrm{~mm}$ e um feixe de $7 \mathrm{~mm}$ do semitendíneo duplo; Rec $\mathrm{M}$, reconstrução de feixe único com um tendão do quadríceps de $10 \mathrm{~mm}$ mais um feixe de $7 \mathrm{~mm}$ do semitendíneo duplo.

conseguiu restaurar a frouxidão do joelho em nenhum ângulo. Diferentes resultados foram obtidos por outros autores, mas com outros métodos de tensionamento e fixação. ${ }^{10,11,13,15}$

Usando um enxerto mais espesso (Rec M), o LDPT não foi significativamente maior do que no joelho intacto a $0^{\circ}, 30^{\circ} \mathrm{e}$ $60^{\circ}$ de flexão. Aos $90^{\circ}$, no entanto, os valores do LDPT foram estatisticamente maiores. Uma vez que a inserção do LCP no
Tabela 5 Comparação dos níveis descritivos do deslocamento posterior da tíbia a $90^{\circ}$ de flexão entre as condições de teste.

\begin{tabular}{|l|l|l|l|l|l|}
\hline & Intacto & Lesionado & $\operatorname{Rec} 1$ & $\operatorname{Rec} 2$ & $\operatorname{Rec} \mathbf{M}$ \\
\hline Intacto & - & $\mathrm{x}$ & $\mathrm{x}$ & $\mathrm{x}$ & $\mathrm{x}$ \\
\hline Lesionado & $p<0,001$ & - & $\mathrm{x}$ & $\mathrm{x}$ & $\mathrm{x}$ \\
\hline $\operatorname{Rec} 1$ & $p<0,001$ & $p<0,001$ & - & $\mathrm{x}$ & $\mathrm{x}$ \\
\hline $\operatorname{Rec} 2$ & $p<0,001$ & $p<0,001$ & $p=0,014$ & - & $\mathrm{x}$ \\
\hline $\operatorname{Rec} \mathrm{M}$ & $p=0,011$ & $p<0,001$ & $p=0,001$ & $p=0,001$ & - \\
\hline
\end{tabular}

Abreviaturas: Intacto, ligamento cruzado posterior intacto; Lesionado, ligamento cruzado posterior lesionado; Rec 1 , reconstrução de feixe único com tendão do quadríceps de $10 \mathrm{~mm}$; $\operatorname{Rec} 2$, reconstrução de duplo feixe com um tendão do quadríceps de $10 \mathrm{~mm}$ e um feixe de $7 \mathrm{~mm}$ do semitendíneo duplo; Rec M, reconstrução de feixe único com um tendão do quadríceps de $10 \mathrm{~mm}$ mais um feixe de $7 \mathrm{~mm}$ do semitendíneo duplo.

fêmur é ampla, correspondendo a 150\% da área de inserção femoral do ligamento cruzado anterior (LCA), e cobrindo cerca de $32 \mathrm{~mm}$ no fêmur, ${ }^{30}$ parece razoável que o enxerto usado para o reconstruir também deva ser mais espesso do que o da reconstrução do LCA. Acreditamos que a melhora com enxerto mais espesso se justifica pela maior quantidade de fibras que se opõem à posteriorização tibial, mais próxima da anatomia normal. Em nossa opinião, os resultados da Rec M melhorariam se pudéssemos preencher todo o espaço femoral do LCP nativo com um enxerto. No entanto, isso não pode ser realizado por túneis cilíndricos. Acreditamos que uma fixação femoral tipo "ïnlay", reproduzindo melhor a inserção anatômica femoral do LCP, poderia ser uma boa opção para se alcançar uma estabilidade cirúrgica ainda melhor.

Em relação à influência do segundo túnel femoral, observamos que ele também melhorou a frouxidão de reconstrução em todos os ângulos testados em comparação com a nossa Rec 1 (tendão do quadríceps de $10 \mathrm{~mm}$ ). Com a Rec 2, o LDPT não apresentou diferenças estatísticas em relação ao joelho com LCP intacto em $0^{\circ}$ e $30^{\circ}$ de flexão. Nos ângulos de $60^{\circ}$ e $90^{\circ}$, no entanto, o LDPT da Rec 2 foi significativamente maior do que o do joelho com LCP intacto. Como o túnel femoral do nosso segundo feixe foi posicionado em uma região mais posterior da inserção anatômica do LCP, ainda mais posterior do que o segundo feixe (PM) descrito por Harner et $a l,{ }^{11}$ e Race e Amis, ${ }^{10}$ e o túnel profundo de Mannor et $\mathrm{al}^{31}$ acreditamos que ele oferecia pouca resistência ao deslocamento posterior da tíbia nesses ângulos maiores de flexão. Em nossa visão, nesses ângulos, o papel-chave foi desempenhado pelo feixe AL, representado pelo enxerto de quadríceps de $10 \mathrm{~mm}$, que, como na Rec 1 , não conseguiu, isoladamente, manter o LDPT próximo ao estado intacto.

Como observado em outros estudos, o acréscimo de mais enxerto através de um segundo túnel femoral também diminuiu a posteriorização da tíbia em nossos testes. ${ }^{2,10,11,18,20,22,23,26}$ Concordamos, todavia, com Bergfeld et $\mathrm{al}^{13}$ que isso não nos permite concluir pela superioridade da Rec 2, pois estamos comparando técnicas com diferentes quantidades de enxerto. Bergfeld et $\mathrm{al}^{13}$ e Kokron et $\mathrm{al}^{12}$ também não encontraram diferenças estatísticas entre a Rec 1 e a $\operatorname{Rec} 2$ ao usar enxertos com a mesma espessura em lesões isoladas do LCP em cadáveres. 
Nesse ponto, a pergunta a ser feita é: já que o aumento da espessura do enxerto melhora a qualidade da reconstrução, é melhor operar com um feixe único mais espesso ou aumentar o volume do enxerto através de um segundo túnel femoral? Para responder a essa questão, comparamos a Rec M com a Rec 2. Em ambas as situações, utilizamos a mesma quantidade de enxerto (tendão do quadríceps, $10 \mathrm{~mm}$, mais semitendíneo duplo, $7 \mathrm{~mm}$ ).

O local onde o enxerto semitendíneo foi adicionado não diferiu em termos do LDPT com o joelho em extensão ou em $30^{\circ}$ de flexão. A colocação do enxerto em um segundo túnel femoral adicional aumentou significativamente o LDPT em comparação com o acréscimo do mesmo enxerto através do mesmo túnel femoral AL. Em outras palavras, a divisão do enxerto em dois feixes agravou a frouxidão da reconstrução em $60^{\circ}$ e $90^{\circ}$ de flexão do joelho.

No campo clínico, três estudos ${ }^{32-34}$ corroboram nossos achados experimentais, relatando melhores resultados com enxertos mais espessos. Essa melhora foi observada tanto nas reconstruções de um quanto nas de dois feixes. Zhao e Huangfu, $^{32}$ em um estudo retrospectivo comparando as técnicas de reconstruções de feixe único, concluíram que o enxerto héptulo oferece mais estabilidade do que o enxerto quádruplo em lesões isoladas do LCP. Isso também foi demonstrado em resultados clínicos de reconstrucões de dois feixes, Zhao et al $^{33}$ publicaram resultados de $88.9 \%$ de pontuação normal e $11.1 \%$ de pontuação quase normal do International Knee Documentation Committee (IKDC) , usando enxerto mais espesso (óctuplo; semitendíneo quádruplo para o feixe AL e grácil quádruplo para o PM). Chen e $\mathrm{Gao}^{34}$ também publicaram resultados com enxerto óctuplo, com 78,9\% de resultados IKDC normais e 15,8\% de quase normais. Portanto, a nosso ver, não há razão para realizar a reconstrução com dois feixes, tecnicamente mais complexa e longa em lesões isoladas do LCP, uma vez que melhores resultados podem ser obtidos pelo acréscimo do enxerto, não através de um segundo túnel femoral, mas através do túnel femoral AL, ou seja, por meio de uma reconstrução com um único feixe mais espesso.

O método utilizado neste estudo permitiu a realização de todas as avaliações em cada joelho. Assim, os resultados de cada técnica poderiam ser comparados entre si e com o comportamento biomecânico do mesmo joelho com o LCP intacto, excluindo qualquer possível viés imposto pelo efeito da variação entre os espécimes. No entanto, para tornar isso possível, a sequência dos testes não pôde ser randomizada, e isso pode ser considerado uma limitação do presente estudo.

\section{Conclusões}

Os resultados deste estudo biomecânico nos permitiram concluir que:

1. Aumentar a espessura do enxerto melhora significativamente a estabilidade da reconstrução do LCP.

2. A divisão do enxerto para a produção dos dois feixes piora a estabilidade da reconstrução do $\mathrm{LCP}$ a $60^{\circ}$ e $90^{\circ}$ de flexão do joelho.
3. Não há justificativa para a realização da reconstrução de dois feixes nas lesões isoladas do LCP, uma vez que a melhora da frouxidão do joelho obtida com essa técnica se deve ao aumento da quantidade de enxerto.

Conflitos de interesse

Os autores declaram não haver conflitos de interesse.

\section{Referências}

1 Harner CD, Höher J. Evaluation and treatment of posterior cruciate ligament injuries. Am J Sports Med 1998;26(03):471-482

2 Wiley WB, Askew MJ, Melby A III, Noe DA. Kinematics of the posterior cruciate ligament/posterolateral corner-injured knee after reconstruction by single- and double-bundle intra-articular grafts. Am J Sports Med 2006;34(05):741-748

3 Kannus P, Bergfeld J, Järvinen $M$, et al. Injuries to the posterior cruciate ligament of the knee. Sports Med 1991;12(02):110-131

4 Parolie JM, Bergfeld JA. Long-term results of nonoperative treatment of isolated posterior cruciate ligament injuries in the athlete. Am J Sports Med 1986;14(01):35-38

5 Dejour H, Walch G, Peyrot J, Eberhard P. Histoire naturelle de la rupture du ligament croisé postérieur. [The natural history of rupture of the posterior cruciate ligament]. Rev Chir Orthop Repar Appar Mot 1988;74(01):35-43

6 Geissler WB, Whipple TL. Intraarticular abnormalities in association with posterior cruciate ligament injuries. Am J Sports Med 1993;21(06):846-849

7 Kohen RB, Sekiya JK. Single-bundle versus double-bundle posterior cruciate ligament reconstruction. Arthroscopy 2009;25(12): 1470-1477

8 Hammoud S, Reinhardt KR, Marx RG. Outcomes of posterior cruciate ligament treatment: a review of the evidence. Sports Med Arthrosc Rev 2010;18(04):280-291

9 Ahmad CS, Cohen ZA, Levine WN, Gardner TR, Ateshian GA, Mow VC. Codominance of the individual posterior cruciate ligament bundles. An analysis of bundle lengths and orientation. Am J Sports Med 2003;31(02):221-225

10 Race A, Amis AA. PCL reconstruction. In vitro biomechanical comparison of 'isometric' versus single and double-bundled 'anatomic' grafts. J Bone Joint Surg Br 1998;80(01):173-179

11 Harner CD, Janaushek MA, Kanamori A, Yagi M, Vogrin TM, Woo SL. Biomechanical analysis of a double-bundle posterior cruciate ligament reconstruction. Am J Sports Med 2000;28(02): 144-151

12 Kokron AWV, Hernandez AJ, Camanho GL, Amatuzzi MM. Biomechanical evaluation of reconstruction laxity with one and two bundles of graft in isolated posterior cruciate ligament injury. In: 2001 International Society of Arthroscopy, Knee Surgery and Orthopedic Sports Medicine - Isakos, 2001. Presentation outlines \& Abstracts. 2001. p. 4-11.

13 Bergfeld JA, Graham SM, Parker RD, Valdevit AD, Kambic HE. A biomechanical comparison of posterior cruciate ligament reconstructions using single- and double-bundle tibial inlay techniques. Am J Sports Med 2005;33(07):976-981

14 Beynnon BD, Amis AA. In vitro testing protocols for the cruciate ligaments and ligament reconstructions. Knee Surg Sports Traumatol Arthrosc 1998;6(Suppl 1):S70-S76

15 Harner CD, Janaushek MA, Ma CB, Kanamori A, Vogrin TM, Woo SL. The effect of knee flexion angle and application of an anterior tibial load at the time of graft fixation on the biomechanics of a posterior cruciate ligament-reconstructed knee. Am J Sports Med 2000;28(04):460-465

16 Ma CB, Kanamori A, Vogrin TM, Woo SL, Harner CD. Measurement of posterior tibial translation in the posterior cruciate ligamentreconstructed knee: significance of the shift in the reference position. Am J Sports Med 2003;31(06):843-848 
17 Chahla J, Moatshe G, Cinque ME, et al. Single-bundle and doublebundle posterior cruciate ligament reconstructions: a systematic review and meta-analysis of 441 patients at a minimum 2 years' follow-up. Arthroscopy 2017;33(11):2066-2080

18 Whiddon DR, Zehms CT, Miller MD, Quinby JS, Montgomery SL, Sekiya JK. Double compared with single-bundle open inlay posterior cruciate ligament reconstruction in a cadaver model. JBone Joint Surg Am 2008;90(09):1820-1829

19 Nuelle CW, Milles JL, Pfeiffer FM, et al. Biomechanical Comparison of Five Posterior Cruciate Ligament Reconstruction Techniques. J Knee Surg 2017;30(06):523-531

20 Milles JL, Nuelle CW, Pfeiffer F, et al. Biomechanical comparison: single-bundle versus double-bundle posterior cruciate ligament reconstruction techniques. J Knee Surg 2017;30(04):347-351

21 Jain V, Goyal A, Mohindra M, Kumar R, Joshi D, Chaudhary D. A comparative analysis of arthroscopic double-bundle versus single-bundle posterior cruciate ligament reconstruction using hamstring tendon autograft. Arch Orthop Trauma Surg 2016; 136(11):1555-1561

22 Wijdicks CA, Kennedy NI, Goldsmith MT, et al. Kinematic analysis of the posterior cruciate ligament, part 2: a comparison of anatomic single- versus double-bundle reconstruction. Am J Sports Med 2013;41(12):2839-2848

23 Li Y, Li J, Wang J, Gao S, Zhang Y. Comparison of single-bundle and double-bundle isolated posterior cruciate ligament reconstruction with allograft: a prospective, randomized study. Arthroscopy 2014;30(06):695-700

24 Xu Y, Yin Y, Wang JQ Ao YF. [Comparison of single and double bundle isolate posterior cruciate ligament reconstruction with hamstring autograft]. Zhonghua Wai Ke Za Zhi 2013;51(03):247-251

25 Yoon KH, Bae DK, Song SJ, Cho HJ, Lee JH. A prospective randomized study comparing arthroscopic single-bundle and doublebundle posterior cruciate ligament reconstructions preserving remnant fibers. Am J Sports Med 2011;39(03):474-480
26 Markolf KL, Feeley BT, Jackson SR, McAllister DR. Biomechanical studies of double-bundle posterior cruciate ligament reconstructions. JBone Joint Surg Am 2006;88(08):1788-1794

27 Apsingi S, Nguyen T, Bull AM, Unwin A, Deehan DJ, Amis AA. Control of laxity in knees with combined posterior cruciate ligament and posterolateral corner deficiency: comparison of single-bundle versus double-bundle posterior cruciate ligament reconstruction combined with modified Larson posterolateral corner reconstruction. Am J Sports Med 2008;36(03):487-494

28 Deie M, Adachi N, Nakamae A, Takazawa K, Ochi M. Evaluation of single-bundle versus double-bundle PCL reconstructions with more than 10-year follow-up. ScientificWorldJournal 2015; 2015:751465

29 Fanelli GC, Beck JD, Edson CJ. Single compared to double-bundle PCL reconstruction using allograft tissue. J Knee Surg 2012;25 (01):59-64

30 Harner CD, Xerogeanes JW, Livesay GA, et al. The human posterior cruciate ligament complex: an interdisciplinary study. Ligament morphology and biomechanical evaluation. Am J Sports Med 1995;23(06):736-745

31 Mannor DA, Shearn JT, Grood ES, Noyes FR, Levy MS. Two-bundle posterior cruciate ligament reconstruction. An in vitro analysis of graft placement and tension. Am J Sports Med 2000;28(06): 833-845

32 Zhao J, Huangfu X. Arthroscopic single-bundle posterior cruciate ligament reconstruction: Retrospective review of 4- versus 7-strand hamstring tendon graft. Knee 2007;14(04):301-305

33 Zhao J, Xiaoqiao H, He Y, Yang X, Liu C, Lu Z. Sandwich-style posterior cruciate ligament reconstruction. Arthroscopy 2008;24 (06):650-659

34 Chen B, Gao S. Double-bundle posterior cruciate ligament reconstruction using a non-hardware suspension fixation technique and 8 strands of autogenous hamstring tendons. Arthroscopy 2009;25 (07):777-782 